\title{
On new generalized quantum integrals and related Hermite-Hadamard inequalities
}

\author{
Hasan Kara', Hüseyin Budak ${ }^{1 *}$, Necmettin Alp ${ }^{1}$, Humaira Kalsoom² and Mehmet Zeki Sarikaya
}

"Correspondence:

hsyn.budak@gmail.com

${ }^{1}$ Department of Mathematics,

Faculty of Science and Arts, Düzce

University, Düzce, Turkey

Full list of author information is

available at the end of the article

\section{Springer}

\begin{abstract}
In this article, we introduce a new concept of quantum integrals which is called ${ }^{\kappa_{2}} T_{q}$-integral. Then we prove several properties of this concept of quantum integrals. Moreover, we present several Hermite-Hadamard type inequalities for ${ }^{{ }_{2}} T_{q}$-integral by utilizing differentiable convex functions. The results presented in this article are unification and generalization of the comparable results in the literature.
\end{abstract}

MSC: 26D10;26D15

Keywords: Hermite-Hadamard's inequalities; Convex functions; q-integrals

\section{Introduction}

In mathematics, the quantum calculus is equivalent to the usual infinitesimal calculus without the concept of limits or the investigation of calculus without limits (quantum is from the Latin word "quantus" and literally it means how much, in Swedish "Kvant"). It has two major branches, $q$-calculus and $h$-calculus. And both of them were worked out by P. Cheung and V. Kac [13] in the early twentieth century. In the same era F.H. Jackson started working on quantum calculus or $q$-calculus, but Euler and Jacobi had already figured out this type of calculus. A number of studies have recently been widely used in the field of $q$-analysis, beginning with Euler, due to the vast necessity for mathematics that models of quantum computing $q$-calculus exist in the framework between physics and mathematics. Tariboon and Ntouyas [19] proposed the quantum calculus concepts on finite intervals and obtained several $q$-analogues of classical mathematical objects. This inspired other researchers and, as a consequence, numerous novel results concerning quantum analogues of classical mathematical results have already been launched in the literature. Noor et al. [14] obtained new $q$-analogues of inequality utilizing first order $q$-differentiable convex function. In [3], Alp et al. acquired some bonds for left-hand side of $q$-Hermite-Hadamard inequalities and quantum calculations by using convex and quasi-convex functions for midpoint form inequalities. For more details, see [13-18, 20] and the references cited therein.

In various mathematical fields, it has many applications, like number theory, combinatorics, orthogonal polynomials, simple hyper-geometric functions, and other sciences, quantum theory, physics and relativity theory; many of the fundamental aspects of quan-

(c) The Author(s) 2021. This article is licensed under a Creative Commons Attribution 4.0 International License, which permits use sharing, adaptation, distribution and reproduction in any medium or format, as long as you give appropriate credit to the original author(s) and the source, provide a link to the Creative Commons licence, and indicate if changes were made. The images or other third party material in this article are included in the article's Creative Commons licence, unless indicated otherwise in a credit line to the material. If material is not included in the article's Creative Commons licence and your intended use is not permitted by statutory regulation or exceeds the permitted use, you will need to obtain permission directly from the copyright holder. To view a copy of this licence, visit http://creativecommons.org/licenses/by/4.0/ 
tum calculus. It has been shown that quantum calculus is a subfield of the more general mathematical field of time scales calculus. New developments have recently been made in the research and methodology of dynamic derivatives on time scales. The research offers a consolidation and application of traditional differential and difference equations. Moreover, it is a unification of the discrete theory with the continuous theory from a theoretical perspective. Time scales provide a unified framework for studying dynamic equations on both discrete and continuous domains. In studying quantum calculus, we are concerned with a specific time scale, called the $q$-time scale, defined as follows: $T:=q^{\mathbb{N}_{0}}:=\left\{q^{t}: t \in \mathbb{N}_{0}\right\}$, see [1-10] and the references cited therein. The HermiteHadamard inequality was introduced by Hermite and Hadamard, see [11]. It is one of the most recognized inequalities in the theory of convex functional analysis, which is stated as follows:

Let $\mathcal{F}:\left[\kappa_{1}, \kappa_{2}\right] \rightarrow \mathbb{R}$ be a convex mapping and $\kappa_{1}<\kappa_{2}$. Then

$$
\mathcal{F}\left(\frac{\kappa_{1}+\kappa_{2}}{2}\right) \leq \frac{1}{\kappa_{2}-\kappa_{1}} \int_{\kappa_{1}}^{\kappa_{2}} \mathcal{F}(\varkappa) d \varkappa \leq \frac{\mathcal{F}\left(\kappa_{1}\right)+\mathcal{F}\left(\kappa_{2}\right)}{2} \text {. }
$$

If $\mathcal{F}$ is concave, both inequalities hold in the reverse direction.

The important purpose of this article is to derive some new quantum integral inequalities of the convex function for a midpoint formula. Moreover, when $q \rightarrow 1$, several examples of Hermite-Hadamard form inequalities are derived as special cases.

\section{Preliminaries of $q$-calculus and some inequalities}

Several fundamental inequalities are well known in classical analysis, like Hölder inequality, Ostrowski inequality, Cauchy-Schwarz inequality, Grüss-Chebyshev inequality, Grüss inequality. Using classical convexity, other basic inequalities have been proven and applied to $q$-calculus. For more details, please see $[2,3,7,9,14,16,18,21]$.

In this section, we discuss some required definitions of quantum calculus and important quantum integral inequalities for Hermite-Hadamard on left and right sides bonds.

$$
[n]_{q}=\frac{1-q^{n}}{1-q}=1+q+q^{2}+\cdots+q^{n-1}, \quad q \in(0,1) .
$$

Jackson derived the $q$-Jackson integral in [12] from 0 to $\kappa_{2}$ for $0<q<1$ as follows:

$$
\int_{0}^{\kappa_{2}} \mathcal{F}(\varkappa) d_{q} \varkappa=(1-q) \kappa_{2} \sum_{n=0}^{\infty} q^{n} \mathcal{F}\left(\kappa_{2} q^{n}\right)
$$

provided the sum converges absolutely.

The $q$-Jackson integral in a generic interval $\left[\kappa_{1}, \kappa_{2}\right]$ was given by in [12] and defined as follows:

$$
\int_{\kappa_{1}}^{\kappa_{2}} \mathcal{F}(\varkappa) d_{q} \varkappa=\int_{0}^{\kappa_{2}} \mathcal{F}(\varkappa) d_{q} \varkappa-\int_{0}^{\kappa_{1}} \mathcal{F}(\varkappa) d_{q} \varkappa
$$

Definition 1 ([19]) We suppose that a function $\mathcal{F}:\left[\kappa_{1}, \kappa_{2}\right] \rightarrow \mathbb{R}$ is continuous. Then the $q_{\kappa_{1}}$-derivative of $\mathcal{F}$ at $\varkappa \in\left[\kappa_{1}, \kappa_{2}\right]$ is defined as follows:

$$
{ }_{\kappa_{1}} D_{q} \mathcal{F}(\varkappa)=\frac{\mathcal{F}(\varkappa)-\mathcal{F}\left(q \varkappa+(1-q) \kappa_{1}\right)}{(1-q)\left(\varkappa-\kappa_{1}\right)}, \quad \varkappa \neq \kappa_{1} .
$$


Since $\mathcal{F}$ is a continuous function from $\left[\kappa_{1}, \kappa_{2}\right]$ to $\mathbb{R}$, so $\kappa_{1} D_{q} \mathcal{F}\left(\kappa_{1}\right)=\lim _{\varkappa \rightarrow \kappa_{1} \kappa_{1}} D_{q} \mathcal{F}(x)$. The function $\mathcal{F}$ is said to be $q$ - differentiable on $\left[\kappa_{1}, \kappa_{2}\right]$ if $\kappa_{1} D_{q} \mathcal{F}(t)$ exists for all $\varkappa \in\left[\kappa_{1}, \kappa_{2}\right]$. If $\kappa_{1}=0$ in (2.2), then ${ }_{0} D_{q} \mathcal{F}(\varkappa)=D_{q} \mathcal{F}(\varkappa)$, where $D_{q} \mathcal{F}(\varkappa)$ is a familiar $q$-derivative of $\mathcal{F}$ at $\varkappa \in\left[\kappa_{1}, \kappa_{2}\right]$ defined by the expression (see [13])

$$
D_{q} \mathcal{F}(\varkappa)=\frac{\mathcal{F}(\varkappa)-\mathcal{F}(q \varkappa)}{(1-q) \varkappa}, \quad \varkappa \neq 0 .
$$

Definition 2 ([5]) We suppose that a function $\mathcal{F}:\left[\kappa_{1}, \kappa_{2}\right] \rightarrow \mathbb{R}$ is continuous, then the $q^{\kappa_{2}}$-derivative of $\mathcal{F}$ at $\varkappa \in\left[\kappa_{1}, \kappa_{2}\right]$ is defined as follows:

$$
{ }^{\kappa_{2}} D_{q} \mathcal{F}(\varkappa)=\frac{\mathcal{F}\left(q \varkappa+(1-q) \kappa_{2}\right)-\mathcal{F}(\varkappa)}{(1-q)\left(\kappa_{2}-\varkappa\right)}, \quad \varkappa \neq \kappa_{2} .
$$

Definition 3 ([19]) We suppose that a function $\mathcal{F}:\left[\kappa_{1}, \kappa_{2}\right] \rightarrow \mathbb{R}$ is continuous, then the $q_{\kappa_{1}}$-definite integral on $\left[\kappa_{1}, \kappa_{2}\right]$ is defined as follows:

$$
\begin{aligned}
\int_{\kappa_{1}}^{\kappa_{2}} \mathcal{F}(\varkappa)_{\kappa_{1}} d_{q} \varkappa & =(1-q)\left(\kappa_{2}-\kappa_{1}\right) \sum_{n=0}^{\infty} q^{n} \mathcal{F}\left(q^{n} \kappa_{2}+\left(1-q^{n}\right) \kappa_{1}\right) \\
& =\left(\kappa_{2}-\kappa_{1}\right) \int_{0}^{1} \mathcal{F}\left((1-t) \kappa_{1}+t \kappa_{2}\right) d_{q} t .
\end{aligned}
$$

In [3], Alp et al. established the $q_{\kappa_{1}}$-Hermite-Hadamard inequalities for convexity, which is defined as follows.

Theorem 1 Let $\mathcal{F}:\left[\kappa_{1}, \kappa_{2}\right] \rightarrow \mathbb{R}$ be a convex differentiable function on $\left[\kappa_{1}, \kappa_{2}\right]$ and $0<q<1$. Then $q$-Hermite-Hadamard inequalities are as follows:

$$
\mathcal{F}\left(\frac{q \kappa_{1}+\kappa_{2}}{1+q}\right) \leq \frac{1}{\kappa_{2}-\kappa_{1}} \int_{\kappa_{1}}^{\kappa_{2}} \mathcal{F}(\varkappa)_{\kappa_{1}} d_{q} \varkappa \leq \frac{q \mathcal{F}\left(\kappa_{1}\right)+\mathcal{F}\left(\kappa_{2}\right)}{1+q}
$$

The authors of [15] and [3] have set certain boundaries for the left and right sides of inequality (2.3).

On the other hand, the following new description and related Hermite-Hadamard form inequalities were given by Bermudo et al.

Definition $4([5])$ Let $\mathcal{F}:\left[\kappa_{1}, \kappa_{2}\right] \rightarrow \mathbb{R}$ be a continuous function. Then the $q^{\kappa_{2}}$-definite integral on $\left[\kappa_{1}, \kappa_{2}\right]$ is defined as

$$
\begin{aligned}
\int_{\kappa_{1}}^{\kappa_{2}} \mathcal{F}(\varkappa)^{\kappa_{2}} d_{q} \varkappa & =(1-q)\left(\kappa_{2}-\kappa_{1}\right) \sum_{n=0}^{\infty} q^{n} \mathcal{F}\left(q^{n} \kappa_{1}+\left(1-q^{n}\right) \kappa_{2}\right) \\
& =\left(\kappa_{2}-\kappa_{1}\right) \int_{0}^{1} \mathcal{F}\left(t \kappa_{1}+(1-t) \kappa_{2}\right) d_{q} t .
\end{aligned}
$$

Theorem $2([5])$ Let $\mathcal{F}:\left[\kappa_{1}, \kappa_{2}\right] \rightarrow \mathbb{R}$ be a convex function on $\left[\kappa_{1}, \kappa_{2}\right]$ and $0<q<1$. Then q-Hermite-Hadamard inequalities are as follows:

$$
\mathcal{F}\left(\frac{\kappa_{1}+q \kappa_{2}}{1+q}\right) \leq \frac{1}{\kappa_{2}-\kappa_{1}} \int_{\kappa_{1}}^{\kappa_{2}} \mathcal{F}(\varkappa)^{\kappa_{2}} d_{q} \varkappa \leq \frac{\mathcal{F}\left(\kappa_{1}\right)+q \mathcal{F}\left(\kappa_{2}\right)}{1+q}
$$


From Theorem 1 and Theorem 2, one can have the following inequalities.

Corollary 1 ([5]) For any convex function $\mathcal{F}:\left[\kappa_{1}, \kappa_{2}\right] \rightarrow \mathbb{R}$ and $0<q<1$, we have

$$
\begin{aligned}
\mathcal{F}\left(\frac{q \kappa_{1}+\kappa_{2}}{1+q}\right)+\mathcal{F}\left(\frac{\kappa_{1}+q \kappa_{2}}{1+q}\right) & \leq \frac{1}{\kappa_{2}-\kappa_{1}}\left\{\int_{\kappa_{1}}^{\kappa_{2}} \mathcal{F}(\varkappa)_{\kappa_{1}} d_{q} \varkappa+\int_{\kappa_{1}}^{\kappa_{2}} \mathcal{F}(\varkappa)^{\kappa_{2}} d_{q} \varkappa\right\} \\
& \leq \mathcal{F}\left(\kappa_{1}\right)+\mathcal{F}\left(\kappa_{2}\right)
\end{aligned}
$$

and

$$
\begin{aligned}
\mathcal{F}\left(\frac{\kappa_{1}+\kappa_{2}}{2}\right) & \leq \frac{1}{2\left(\kappa_{2}-\kappa_{1}\right)}\left\{\int_{\kappa_{1}}^{\kappa_{2}} \mathcal{F}(\varkappa)_{\kappa_{1}} d_{q} \varkappa+\int_{\kappa_{1}}^{\kappa_{2}} \mathcal{F}(\varkappa)^{\kappa_{2}} d_{q} \varkappa\right\} \\
& \leq \frac{\mathcal{F}\left(\kappa_{1}\right)+\mathcal{F}\left(\kappa_{2}\right)}{2} .
\end{aligned}
$$

Alp and Sarikaya, by using the area of trapezoids, introduced the following generalized quantum integral which we will call ${ }_{\kappa_{1}} T_{q}$-integral.

Definition $5([1])$ Let $\mathcal{F}:\left[\kappa_{1}, \kappa_{2}\right] \rightarrow \mathbb{R}$ be a continuous function. For $\varkappa \in\left[\kappa_{1}, \kappa_{2}\right]$,

$$
\int_{\kappa_{1}}^{\kappa_{2}} \mathcal{F}(\xi)_{\kappa_{1}} d_{q}^{T} \xi=\frac{(1-q)\left(\kappa_{2}-\kappa_{1}\right)}{2 q}\left[(1+q) \sum_{n=0}^{\infty} q^{n} \mathcal{F}\left(q^{n} \kappa_{2}+\left(1-q^{n}\right) \kappa_{1}\right)-\mathcal{F}\left(\kappa_{2}\right)\right]
$$

where $0<q<1$.

Theorem $3\left({ }_{\kappa_{1}} T_{q}\right.$-Hermite-Hadamard, [1]) Let $\mathcal{F}:\left[\kappa_{1}, \kappa_{2}\right] \rightarrow \mathbb{R}$ be a convex continuous function on $\left[\kappa_{1}, \kappa_{2}\right]$ and $0<q<1$. Then we have

$$
\mathcal{F}\left(\frac{\kappa_{1}+\kappa_{2}}{2}\right) \leq \frac{1}{\kappa_{2}-\kappa_{1}} \int_{\kappa_{1}}^{\kappa_{2}} \mathcal{F}(\varkappa)_{\kappa_{1}} d_{q}^{T} \varkappa \leq \frac{\mathcal{F}\left(\kappa_{1}\right)+\mathcal{F}\left(\kappa_{2}\right)}{2}
$$

\section{New generalized quantum integrals}

In this section, we introduce a new generalized quantum integral which is called ${ }^{\kappa_{2}} T_{q^{-}}$ integral. We also prove several properties of this integral.

As can be seen from Fig. 1 , the area of $n$ th-trapezoid is

$$
B_{n}=(1-q) q^{n}\left(\kappa_{2}-\kappa_{1}\right) \frac{\mathcal{F}\left(q^{n+1} \kappa_{1}+\left(1-q^{n+1}\right) \kappa_{2}\right)+\mathcal{F}\left(q^{n} \kappa_{1}+\left(1-q^{n}\right) \kappa_{2}\right)}{2} .
$$

By summing all the area of $B_{n}, n=1,2, \ldots$, we have

$$
\begin{aligned}
\sum_{n=0}^{\infty} B_{n}= & \frac{(1-q)\left(\kappa_{2}-\kappa_{1}\right)}{2}\left[\sum_{n=0}^{\infty} q^{n} \mathcal{F}\left(q^{n+1} \kappa_{1}+\left(1-q^{n+1}\right) \kappa_{2}\right)\right. \\
& \left.+\sum_{n=0}^{\infty} q^{n} \mathcal{F}\left(q^{n} \kappa_{1}+\left(1-q^{n}\right) \kappa_{2}\right)\right]
\end{aligned}
$$




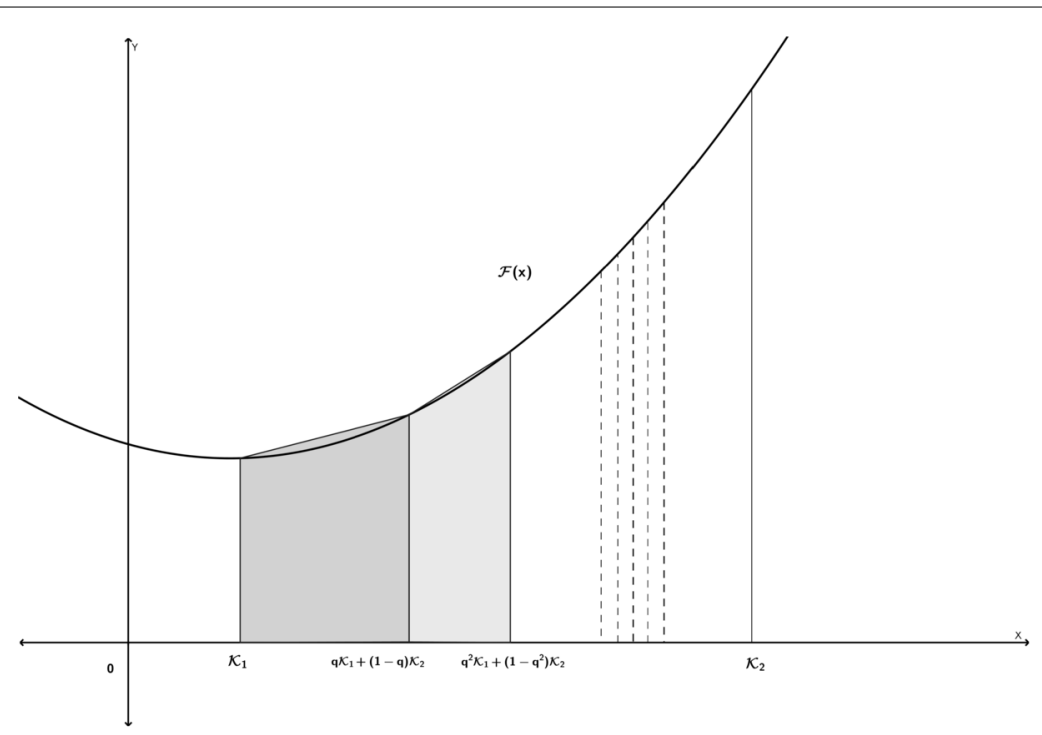

Figure 1 Areas of trapezoids

$$
\begin{aligned}
&= \frac{(1-q)\left(\kappa_{2}-\kappa_{1}\right)}{2}\left[\frac{1}{q} \sum_{n=0}^{\infty} q^{n+1} \mathcal{F}\left(q^{n+1} \kappa_{1}+\left(1-q^{n+1}\right) \kappa_{2}\right)\right. \\
&\left.+\sum_{n=0}^{\infty} q^{n} \mathcal{F}\left(q^{n} \kappa_{1}+\left(1-q^{n}\right) \kappa_{2}\right)\right] \\
&= \frac{(1-q)\left(\kappa_{2}-\kappa_{1}\right)}{2}\left[\frac{1}{q} \sum_{n=1}^{\infty} q^{n} \mathcal{F}\left(q^{n} \kappa_{1}+\left(1-q^{n}\right) \kappa_{2}\right)\right. \\
&\left.+\sum_{n=0}^{\infty} q^{n} \mathcal{F}\left(q^{n} \kappa_{1}+\left(1-q^{n}\right) \kappa_{2}\right)\right] \\
&= \frac{(1-q)\left(\kappa_{2}-\kappa_{1}\right)}{2}\left[\frac{1}{q}\left\{\mathcal{F}\left(\kappa_{1}\right)-\mathcal{F}\left(\kappa_{1}\right)+\sum_{n=1}^{\infty} q^{n} \mathcal{F}\left(q^{n} \kappa_{1}+\left(1-q^{n}\right) \kappa_{2}\right)\right\}\right. \\
&\left.+\sum_{n=0}^{\infty} q^{n} \mathcal{F}\left(q^{n} \kappa_{1}+\left(1-q^{n}\right) \kappa_{2}\right)\right] \\
&= \frac{(1-q)\left(\kappa_{2}-\kappa_{1}\right)}{2}\left[\frac{1}{q}\left\{-\mathcal{F}\left(\kappa_{1}\right)+\sum_{n=0}^{\infty} q^{n} \mathcal{F}\left(q^{n} \kappa_{1}+\left(1-q^{n}\right) \kappa_{2}\right)\right\}\right. \\
&\left.+\sum_{n=0}^{\infty} q^{n} \mathcal{F}\left(q^{n} \kappa_{1}+\left(1-q^{n}\right) \kappa_{2}\right)\right] \\
&= \frac{(1-q)\left(\kappa_{2}-\kappa_{1}\right)}{2 q}\left[(1+q) \sum_{n=0}^{\infty} q^{n} \mathcal{F}\left(q^{n} \kappa_{1}+\left(1-q^{n}\right) \kappa_{2}\right)-\mathcal{F}\left(\kappa_{1}\right)\right] \\
& \kappa_{1}(\xi)^{\kappa_{2}} d_{q}^{T} \xi . \\
&
\end{aligned}
$$

Now we can give the following definition. 
Definition 6 Let $\mathcal{F}:\left[\kappa_{1}, \kappa_{2}\right] \rightarrow \mathbb{R}$ be a continuous function. For $\varkappa \in\left[\kappa_{1}, \kappa_{2}\right]$,

$$
\int_{\kappa_{1}}^{\kappa_{2}} \mathcal{F}(\xi)^{\kappa_{2}} d_{q}^{T} \xi=\frac{(1-q)\left(\kappa_{2}-\kappa_{1}\right)}{2 q}\left[(1+q) \sum_{n=0}^{\infty} q^{n} \mathcal{F}\left(q^{n} \kappa_{1}+\left(1-q^{n}\right) \kappa_{2}\right)-\mathcal{F}\left(\kappa_{1}\right)\right]
$$

where $0<q<1$. This integral is called ${ }^{\kappa_{2}} T_{q}$-integral.

Theorem 4 Let $\mathcal{F}:\left[\kappa_{1}, \kappa_{2}\right] \rightarrow \mathbb{R}$ be a continuous function. Then we have

$$
{ }^{\kappa_{2}} D_{q} \int_{\varkappa}^{\kappa_{2}} \mathcal{F}(\xi){ }^{\kappa_{2}} d_{q}^{T} \xi=-\frac{\mathcal{F}(\varkappa)+\mathcal{F}\left(q \varkappa+(1-q) \kappa_{2}\right)}{2}
$$

for $\varkappa \in\left[\kappa_{1}, \kappa_{2}\right]$.

Proof From the definition of ${ }^{\kappa_{2}} T_{q}$-integral, we have

$$
\int_{\varkappa}^{\kappa_{2}} \mathcal{F}(\xi)^{\kappa_{2}} d_{q}^{T} \xi=\frac{(1-q)\left(\kappa_{2}-\varkappa\right)}{2 q}\left[(1+q) \sum_{n=0}^{\infty} q^{n} \mathcal{F}\left(q^{n} \varkappa+\left(1-q^{n}\right) \kappa_{2}\right)-\mathcal{F}(\varkappa)\right] .
$$

From Definition 2, we obtain

$$
\begin{aligned}
{ }^{\kappa_{2}} & D_{q} \int_{\varkappa}^{\kappa_{2}} \mathcal{F}(\xi)^{\kappa_{2}} d_{q}^{T} \xi \\
= & { }^{{ }_{2}} D_{q}\left\{\frac{(1-q)\left(\kappa_{2}-\varkappa\right)}{2 q}\left[(1+q) \sum_{n=0}^{\infty} q^{n} \mathcal{F}\left(q^{n} \varkappa+\left(1-q^{n}\right) \kappa_{2}\right)-\mathcal{F}(\varkappa)\right]\right\} \\
= & \frac{1}{(1-q)\left(\kappa_{2}-\varkappa\right)}\left\{\frac{(1-q)\left(\kappa_{2}-\varkappa\right) q}{2 q}\right. \\
& \times\left[(1+q) \sum_{n=0}^{\infty} q^{n} \mathcal{F}\left(q^{n+1} \varkappa+\left(1-q^{n+1}\right) \kappa_{2}\right)-\mathcal{F}\left(q \varkappa+(1-q) \kappa_{2}\right)\right] \\
& \left.-\frac{(1-q)\left(\kappa_{2}-\varkappa\right)}{2 q}\left[(1+q) \sum_{n=0}^{\infty} q^{n} \mathcal{F}\left(q^{n} \varkappa+\left(1-q^{n}\right) \kappa_{2}\right)-\mathcal{F}(\varkappa)\right]\right\} \\
= & \frac{1}{2 q}\left[(1+q)\left(q \sum_{n=0}^{\infty} q^{n} \mathcal{F}\left(q^{n+1} \varkappa+\left(1-q^{n+1}\right) \kappa_{2}\right)-\sum_{n=0}^{\infty} q^{n} \mathcal{F}\left(q^{n} \varkappa+\left(1-q^{n}\right) \kappa_{2}\right)\right)\right. \\
& \left.\times \mathcal{F}(\varkappa)-q \mathcal{F}\left(q \varkappa+(1-q) \kappa_{2}\right)\right] \\
= & -\frac{\mathcal{F}(\varkappa)+\mathcal{F}\left(q \varkappa+(1-q) \kappa_{2}\right)}{2} .
\end{aligned}
$$

The proof is completed.

Theorem 5 Let $\mathcal{F}:\left[\kappa_{1}, \kappa_{2}\right] \rightarrow \mathbb{R}$ be a function and $0<q<1$. Then we have

$$
\int_{0}^{1} \mathcal{F}\left(\xi \kappa_{2}+(1-\xi) \kappa_{1}\right)^{1} d_{q}^{T} \xi=\frac{1}{\kappa_{2}-\kappa_{1}} \int_{\kappa_{1}}^{\kappa_{2}} \mathcal{F}(t)^{\kappa_{2}} d_{q}^{T} t
$$


Proof From the definition of ${ }^{\kappa_{2}} T_{q}$-integral, we have

$$
\begin{array}{rl}
\int_{0}^{1} & \mathcal{F}\left(\xi \kappa_{2}+(1-\xi) \kappa_{1}\right)^{1} d_{q}^{T} \xi \\
= & \frac{(1-q)(1-0)}{2 q}\left[(1+q) \sum_{n=0}^{\infty} q^{n} \mathcal{F}\left(\left[q^{n} 0+\left(1-q^{n}\right) 1\right] \kappa_{2}+\left(1-\left[q^{n} 0+\left(1-q^{n}\right) 1\right]\right) \kappa_{1}\right)\right. \\
& \left.-\mathcal{F}\left(0 b+(1-0) \kappa_{1}\right)\right] \\
= & \frac{(1-q)}{2 q}\left[(1+q) \sum_{n=0}^{\infty} q^{n} \mathcal{F}\left(q^{n} \kappa_{1}+\left(1-q^{n}\right) \kappa_{2}\right)-\mathcal{F}\left(\kappa_{1}\right)\right] \\
= & \frac{1}{\kappa_{2}-\kappa_{1}} \int_{\kappa_{1}}^{\kappa_{2}} \mathcal{F}(t)^{\kappa_{2}} d_{q}^{T} t .
\end{array}
$$

The proof is completed.

Theorem 6 Let $\mathcal{F}:\left[\kappa_{1}, \kappa_{2}\right] \rightarrow \mathbb{R}$ be a continuous function. Then we have

$$
\int_{\varkappa}^{\kappa_{2}}{ }^{\kappa_{2}} D_{q} \mathcal{F}(\xi){ }^{\kappa_{2}} d_{q}^{T} \xi=\frac{(1+q) \mathcal{F}\left(\kappa_{2}\right)-q \mathcal{F}(\varkappa)-\mathcal{F}\left(q \varkappa+(1-q) \kappa_{2}\right)}{2 q}
$$

for $\varkappa \in\left(\kappa_{1}, \kappa_{2}\right)$.

Proof From Definition 2, we have

$$
{ }_{\kappa_{2}} D_{q} \mathcal{F}(\xi)=\frac{\mathcal{F}\left(q \xi+(1-q) \kappa_{2}\right)-\mathcal{F}(\xi)}{(1-q)\left(\kappa_{2}-\xi\right)}
$$

By using Definition 6, we have

$$
\begin{aligned}
\int_{\varkappa}^{\kappa_{2}} & { }_{2} D_{q} \mathcal{F}(\xi)^{\kappa_{2}} d_{q}^{T} \xi \\
= & \int_{\varkappa}^{\kappa_{2}} \frac{\mathcal{F}\left(q \xi+(1-q) \kappa_{2}\right)-\mathcal{F}(\xi)}{(1-q)\left(\kappa_{2}-\xi\right)} \kappa_{2} d_{q}^{T} \xi \\
= & \frac{(1-q)\left(\kappa_{2}-\varkappa\right)}{2 q}\left[(1+q) \sum_{n=0}^{\infty} \frac{q^{n} \mathcal{F}\left(q^{n+1} \varkappa+\left(1-q^{n+1}\right) \kappa_{2}\right)}{(1-q) q^{n}\left(\kappa_{2}-\varkappa\right)}-\frac{\mathcal{F}\left(q \varkappa+(1-q) \kappa_{2}\right)}{(1-q)\left(\kappa_{2}-\varkappa\right)}\right] \\
& -\frac{(1-q)\left(\kappa_{2}-\varkappa\right)}{2 q}\left[(1+q) \sum_{n=0}^{\infty} \frac{q^{n} \mathcal{F}\left(q^{n} \varkappa+\left(1-q^{n}\right) \kappa_{2}\right)}{(1-q) q^{n}\left(\kappa_{2}-\varkappa\right)}-\frac{\mathcal{F}(\varkappa)}{(1-q)\left(\kappa_{2}-\varkappa\right)}\right] \\
= & \frac{1+q}{2 q} \sum_{n=0}^{\infty}\left[\mathcal{F}\left(q^{n+1} \varkappa+\left(1-q^{n+1}\right) \kappa_{2}\right)-\mathcal{F}\left(q^{n} \varkappa+\left(1-q^{n}\right) \kappa_{2}\right)\right] \\
& +\frac{1}{2 q}\left[\mathcal{F}\left(q \varkappa+(1-q) \kappa_{2}\right)+\mathcal{F}(\varkappa)\right] \\
= & \frac{(1+q) \mathcal{F}\left(\kappa_{2}\right)-q \mathcal{F}(\varkappa)-\mathcal{F}\left(q \varkappa+(1-q) \kappa_{2}\right)}{2 q} .
\end{aligned}
$$

The proof is completed. 
Theorem 7 Assume that $\mathcal{F}, g:\left[\kappa_{1}, \kappa_{2}\right] \rightarrow \mathbb{R}$ are continuous functions. Then we have

$$
\int_{\varkappa}^{\kappa_{2}}[\mathcal{F}(\xi)+g(\xi)]^{\kappa_{2}} d_{q}^{T} \xi=\int_{\varkappa}^{\kappa_{2}} \mathcal{F}(\xi)^{\kappa_{2}} d_{q}^{T} \xi+\int_{\varkappa}^{\kappa_{2}} g(\xi)^{\kappa_{2}} d_{q}^{T} \xi
$$

for $\varkappa \in\left[\kappa_{1}, \kappa_{2}\right]$.

Proof Using the definition of ${ }^{\kappa_{2}} T_{q}$-integral, we can write that

$$
\begin{aligned}
& \int_{\varkappa}^{\kappa_{2}}[\mathcal{F}(\xi)+g(\xi)]^{\kappa_{2}} d_{q}^{T} \xi \\
&=\frac{(1-q)\left(\kappa_{2}-\varkappa\right)}{2 q}\left\{(1+q) \sum_{n=0}^{\infty} q^{n}\left[\mathcal{F}\left(q^{n} \varkappa+\left(1-q^{n}\right) \kappa_{2}\right)+g\left(q^{n} \varkappa+\left(1-q^{n}\right) \kappa_{2}\right)\right]\right. \\
&-\mathcal{F}(\varkappa)-g(\varkappa)\} \\
&= \frac{(1-q)\left(\kappa_{2}-\varkappa\right)}{2 q}\left[(1+q) \sum_{n=0}^{\infty} q^{n} \mathcal{F}\left(q^{n} \varkappa+\left(1-q^{n}\right) \kappa_{2}\right)-\mathcal{F}(\varkappa)\right] \\
&+\frac{(1-q)\left(\kappa_{2}-\varkappa\right)}{2 q}\left[(1+q) \sum_{n=0}^{\infty} q^{n} g\left(q^{n} \varkappa+\left(1-q^{n}\right) \kappa_{2}\right)-g(\varkappa)\right] \\
&= \int_{\varkappa}^{\kappa_{2}} \mathcal{F}(\xi)^{\kappa_{2}} d_{q}^{T} \xi+\int_{\varkappa}^{\kappa_{2}} g(\xi)^{\kappa_{2}} d_{q}^{T} \xi
\end{aligned}
$$

which finishes proof.

Theorem 8 Assume that $\mathcal{F}:\left[\kappa_{1}, \kappa_{2}\right] \rightarrow \mathbb{R}$ is a continuous function and $\alpha \in \mathbb{R}$. Then

$$
\int_{\varkappa}^{\kappa_{2}}(\alpha \mathcal{F})(\xi)^{\kappa_{2}} d_{q}^{T} \xi=\alpha \int_{\varkappa}^{\kappa_{2}} \mathcal{F}(\xi)^{\kappa_{2}} d_{q}^{T} \xi
$$

for $\varkappa \in\left[\kappa_{1}, \kappa_{2}\right]$.

Proof By the definition of ${ }^{\kappa_{2}} T_{q}$-integral, we have

$$
\begin{aligned}
& \int_{\varkappa}^{\kappa_{2}}(\alpha \mathcal{F})(\xi)^{\kappa_{2}} d_{q}^{T} \xi \\
& =\frac{(1-q)\left(\kappa_{2}-\varkappa\right)}{2 q}\left[(1+q) \sum_{n=0}^{\infty} q^{n}(\alpha \mathcal{F})\left(q^{n} \varkappa+\left(1-q^{n}\right) \kappa_{2}\right)-(\alpha \mathcal{F})(\varkappa)\right] \\
& =\alpha \frac{(1-q)\left(\kappa_{2}-\varkappa\right)}{2 q}\left[(1+q) \sum_{n=0}^{\infty} q^{n} \mathcal{F}\left(q^{n} \varkappa+\left(1-q^{n}\right) \kappa_{2}\right)-\mathcal{F}(\varkappa)\right] \\
& =\alpha \int_{\varkappa}^{\kappa_{2}} \mathcal{F}(\xi)^{\kappa_{2}} d_{q}^{T} \xi
\end{aligned}
$$


Theorem 9 Assume that $\mathcal{F}, g:\left[\kappa_{1}, \kappa_{2}\right] \rightarrow \mathbb{R}$ are continuous functions. Then we have

$$
\begin{array}{rl}
\int_{\varkappa}^{\kappa_{2}} & \mathcal{F}(\xi)^{\kappa_{2}} D_{q} g(\xi)^{\kappa_{2}} d_{q}^{T} \xi \\
= & \left.\frac{q \mathcal{F}(\xi) g(\xi)+\mathcal{F}\left(q \xi+(1-q) \kappa_{2}\right) g\left(q \xi+(1-q) \kappa_{2}\right)}{2 q}\right|_{\varkappa} ^{\kappa_{2}} \\
& -\int_{\varkappa}^{\kappa_{2}} g\left(q \xi+(1-q) \kappa_{2}\right)^{\kappa_{2}} D_{q} \mathcal{F}(\xi)^{\kappa_{2}} d_{q}^{T} \xi
\end{array}
$$

for $\varkappa \in\left[\kappa_{1}, \kappa_{2}\right]$.

Proof Using Definition 2, we get

$$
\begin{aligned}
& { }^{\kappa_{2}} D_{q}(\mathcal{F}(\xi) g(\xi)) \\
& \quad=\frac{\mathcal{F}\left(q \xi+(1-q) \kappa_{2}\right) g\left(q \xi+(1-q) \kappa_{2}\right)-\mathcal{F}(\xi) g(\xi)}{(1-q)\left(\kappa_{2}-\xi\right)} \\
& \quad=\mathcal{F}(\xi) \frac{g\left(q \xi+(1-q) \kappa_{2}\right)-g(\xi)}{(1-q)\left(\kappa_{2}-\xi\right)}+g\left(q \xi+(1-q) \kappa_{2}\right) \frac{\mathcal{F}\left(q \xi+(1-q) \kappa_{2}\right)-\mathcal{F}(\xi)}{(1-q)\left(\kappa_{2}-\xi\right)} \\
& \quad=\mathcal{F}(\xi){ }^{\kappa_{2}} D_{q} g(\xi)+g\left(q \xi+(1-q) \kappa_{2}\right){ }^{\kappa_{2}} D_{q} \mathcal{F}(\xi) .
\end{aligned}
$$

By taking the ${ }^{\kappa_{2}} T_{q}$-integral of equality (3.8), we get

$$
\begin{aligned}
& \int_{\varkappa}^{\kappa_{2}}{ }^{\kappa_{2}} D_{q}(\mathcal{F}(\xi) g(\xi))^{\kappa_{2}} d_{q}^{T} \xi \\
& \quad=\int_{\varkappa}^{\kappa_{2}} \mathcal{F}(\xi)^{\kappa_{2}} D_{q} g(\xi)^{\kappa_{2}} d_{q}^{T} \xi+\int_{\varkappa}^{\kappa_{2}} g\left(q \xi+(1-q) \kappa_{2}\right){ }^{\kappa_{2}} D_{q} \mathcal{F}(\xi)^{\kappa_{2}} d_{q}^{T} \xi
\end{aligned}
$$

By applying Theorem 6, we have

$$
\begin{aligned}
& \int_{\varkappa}^{\kappa_{2}}{ }^{\kappa_{2}} D_{q}(\mathcal{F}(\xi) g(\xi)){ }^{\kappa_{2}} d_{q}^{T} \xi \\
& =\frac{(1+q) \mathcal{F}\left(\kappa_{2}\right) g\left(\kappa_{2}\right)-q \mathcal{F}(\xi) g(\xi)-\mathcal{F}\left(q \xi+(1-q) \kappa_{2}\right) g\left(q \xi+(1-q) \kappa_{2}\right)}{2 q} \\
& =\left.\frac{q \mathcal{F}(\xi) g(\xi)+\mathcal{F}\left(q \xi+(1-q) \kappa_{2}\right) g\left(q \xi+(1-q) \kappa_{2}\right)}{2 q}\right|_{\varkappa} ^{\kappa_{2}} .
\end{aligned}
$$

From equalities (3.9) and (3.10), we obtain

$$
\begin{aligned}
\int_{\varkappa}^{\kappa_{2}} \mathcal{F}(\xi)^{\kappa_{2}} D_{q} g(\xi)^{\kappa_{2}} d_{q}^{T} \xi= & \left.\frac{q \mathcal{F}(\xi) g(\xi)+\mathcal{F}\left(q \xi+(1-q) \kappa_{2}\right) g\left(q \xi+(1-q) \kappa_{2}\right)}{2 q}\right|_{\varkappa} ^{\kappa_{2}} \\
& -\int_{\varkappa}^{\kappa_{2}} g\left(q \xi+(1-q) \kappa_{2}\right){ }^{\kappa_{2}} D_{q} \mathcal{F}(\xi)^{\kappa_{2}} d_{q}^{T} \xi .
\end{aligned}
$$

This completes the proof. 
Theorem 10 Assume that $\mathcal{F}, g:\left[\kappa_{1}, \kappa_{2}\right] \rightarrow \mathbb{R}$ are continuous functions and $\mathcal{F}(\xi) \leq g(\xi)$ for all $\xi \in\left[\varkappa, \kappa_{2}\right]$. Then we have

$$
\int_{\varkappa}^{\kappa_{2}} \mathcal{F}(\xi)^{\kappa_{2}} d_{q}^{T} \xi \leq \int_{\varkappa}^{\kappa_{2}} g(\xi)^{\kappa_{2}} d_{q}^{T} \xi
$$

for $\varkappa \in\left[\kappa_{1}, \kappa_{2}\right]$.

Proof By the definition of ${ }^{\kappa_{2}} T_{q}$-integral, we have

$$
\begin{aligned}
\int_{\varkappa}^{\kappa_{2}} \mathcal{F}(\xi)^{\kappa_{2}} d_{q}^{T} \xi & =\frac{(1-q)\left(\kappa_{2}-\varkappa\right)}{2 q}\left[(1+q) \sum_{n=0}^{\infty} q^{n} \mathcal{F}\left(q^{n} \varkappa+\left(1-q^{n}\right) \kappa_{2}\right)-\mathcal{F}(\varkappa)\right] \\
& =\frac{(1-q)\left(\kappa_{2}-\varkappa\right)}{2 q}\left[(1+q) \sum_{n=1}^{\infty} q^{n} \mathcal{F}\left(q^{n} \varkappa+\left(1-q^{n}\right) \kappa_{2}\right)+q \mathcal{F}(\varkappa)\right] \\
& \leq \frac{(1-q)\left(\kappa_{2}-\varkappa\right)}{2 q}\left[(1+q) \sum_{n=1}^{\infty} q^{n} g\left(q^{n} \varkappa+\left(1-q^{n}\right) \kappa_{2}\right)+q g(\varkappa)\right] \\
& =\frac{(1-q)\left(\kappa_{2}-\varkappa\right)}{2 q}\left[(1+q) \sum_{n=0}^{\infty} q^{n} g\left(q^{n} \varkappa+\left(1-q^{n}\right) \kappa_{2}\right)-g(\varkappa)\right] \\
& =\int_{\varkappa}^{\kappa_{2}} g(\xi)^{\kappa_{2}} d_{q}^{T} \xi .
\end{aligned}
$$

Proposition 1 For $\alpha \in \mathbb{R} \backslash\{-1\}$, we have the following equality:

$$
\int_{x}^{b}(b-s)^{\alpha b} d_{q}^{T} s=\frac{1+q^{\alpha}}{2[\alpha+1]_{q}}(b-x)^{\alpha+1} .
$$

Proof Using the definition of ${ }^{\kappa_{2}} T_{q}$-integral, we have

$$
\begin{aligned}
\int_{\varkappa}^{\kappa_{2}} & \left(\kappa_{2}-\xi\right)^{\alpha \kappa_{2}} d_{q}^{T} \xi \\
= & \frac{(1-q)\left(\kappa_{2}-\varkappa\right)}{2 q}\left[(1+q) \sum_{n=0}^{\infty} q^{n}\left(\kappa_{2}-\left(q^{n} \varkappa+\left(1-q^{n}\right) \kappa_{2}\right)\right)^{\alpha}-\left(\kappa_{2}-\varkappa\right)^{\alpha}\right] \\
= & \frac{(1-q)\left(\varkappa-\kappa_{1}\right)}{2 q}\left[(1+q) \sum_{n=0}^{\infty} q^{n}\left(q^{n}\left(\kappa_{2}-\varkappa\right)\right)^{\alpha}-\left(\kappa_{2}-\varkappa\right)^{\alpha}\right] \\
= & \frac{(1-q)\left(\kappa_{2}-\varkappa\right)}{2 q}\left[(1+q)\left(\kappa_{2}-\varkappa\right)^{\alpha} \sum_{n=0}^{\infty}\left(q^{\alpha+1}\right)^{n}-\left(\kappa_{2}-\varkappa\right)^{\alpha}\right] \\
= & \frac{(1-q)\left(\kappa_{2}-\varkappa\right)^{\alpha+1}}{2 q}\left[\frac{(1+q)}{1-q^{\alpha+1}}-1\right] \\
= & \frac{1-q}{1-q^{\alpha+1}} \frac{1+q^{\alpha}}{2}\left(\kappa_{2}-\varkappa\right)^{\alpha+1} \\
= & \frac{1}{[\alpha+1]_{q}} \frac{1+q^{\alpha}}{2}\left(\kappa_{2}-\varkappa\right)^{\alpha+1} .
\end{aligned}
$$

The proof is completed. 
Kara et al. Journal of Inequalities and Applications

(2021) 2021:180

Page 11 of 15

4 Hermite-Hadamard inequalities for ${ }^{\kappa_{2}} T_{q}$-integral

In this section, we present some Hermite-Hadamard type inequalities for ${ }^{\kappa_{2}} T_{q}$-integral by utilizing convex functions.

Theorem 11 Let $\mathcal{F}:\left[\kappa_{1}, \kappa_{2}\right] \rightarrow \mathbb{R}$ be a convex continuous function on $\left[\kappa_{1}, \kappa_{2}\right]$ and $0<q<1$.

Then we have

$$
\mathcal{F}\left(\frac{\kappa_{1}+\kappa_{2}}{2}\right) \leq \frac{1}{\kappa_{2}-\kappa_{1}} \int_{\kappa_{1}}^{\kappa_{2}} \mathcal{F}(\varkappa)^{\kappa_{2}} d_{q}^{T} \varkappa \leq \frac{\mathcal{F}\left(\kappa_{1}\right)+\mathcal{F}\left(\kappa_{2}\right)}{2} .
$$

Proof Since $\mathcal{F}$ is a differentiable function on $\left[\kappa_{1}, \kappa_{2}\right]$, there is a tangent line for the function $\mathcal{F}$ at the point $\frac{\kappa_{1}+\kappa_{2}}{2} \in\left(\kappa_{1}, \kappa_{2}\right)$. This tangent line can be expressed as a function $\Psi_{1}(\varkappa)=$ $\mathcal{F}\left(\frac{\kappa_{1}+\kappa_{2}}{2}\right)+\mathcal{F}^{\prime}\left(\frac{\kappa_{1}+\kappa_{2}}{2}\right)\left(\varkappa-\frac{\kappa_{1}+\kappa_{2}}{2}\right)$.

Since $\mathcal{F}$ is a convex function on $\left[\kappa_{1}, \kappa_{2}\right]$, then we have the following inequality:

$$
\Psi_{1}(\varkappa)=\mathcal{F}\left(\frac{\kappa_{1}+\kappa_{2}}{2}\right)+\mathcal{F}^{\prime}\left(\frac{\kappa_{1}+\kappa_{2}}{2}\right)\left(\varkappa-\frac{\kappa_{1}+\kappa_{2}}{2}\right) \leq \mathcal{F}(\varkappa)
$$

for all $\varkappa \in\left[\kappa_{1}, \kappa_{2}\right]$ (see Fig. 2). From Theorem 10, we have

$$
\int_{\kappa_{1}}^{\kappa_{2}} \Psi_{1}(\varkappa)^{\kappa_{2}} d_{q}^{T} \varkappa \leq \int_{\kappa_{1}}^{\kappa_{2}} \mathcal{F}(\varkappa)^{\kappa_{2}} d_{q}^{T} \varkappa
$$

By Definition 6, we have

$$
\begin{aligned}
& \int_{\kappa_{1}}^{\kappa_{2}} \Psi_{1}(\varkappa)^{\kappa_{2}} d_{q}^{T} \varkappa \\
& =\int_{\kappa_{1}}^{\kappa_{2}}\left[\mathcal{F}\left(\frac{\kappa_{1}+\kappa_{2}}{2}\right)+\mathcal{F}^{\prime}\left(\frac{\kappa_{1}+\kappa_{2}}{2}\right)\left(\varkappa-\frac{\kappa_{1}+\kappa_{2}}{2}\right)\right] \kappa_{2} d_{q}^{T} \varkappa \\
& =\left(\kappa_{2}-\kappa_{1}\right) \mathcal{F}\left(\frac{\kappa_{1}+\kappa_{2}}{2}\right)-\mathcal{F}^{\prime}\left(\frac{\kappa_{1}+\kappa_{2}}{2}\right) \int_{\kappa_{1}}^{\kappa_{2}}\left(\kappa_{2}-\varkappa+\frac{\kappa_{1}-\kappa_{2}}{2}\right) \kappa_{2} d_{q}^{T} \varkappa
\end{aligned}
$$

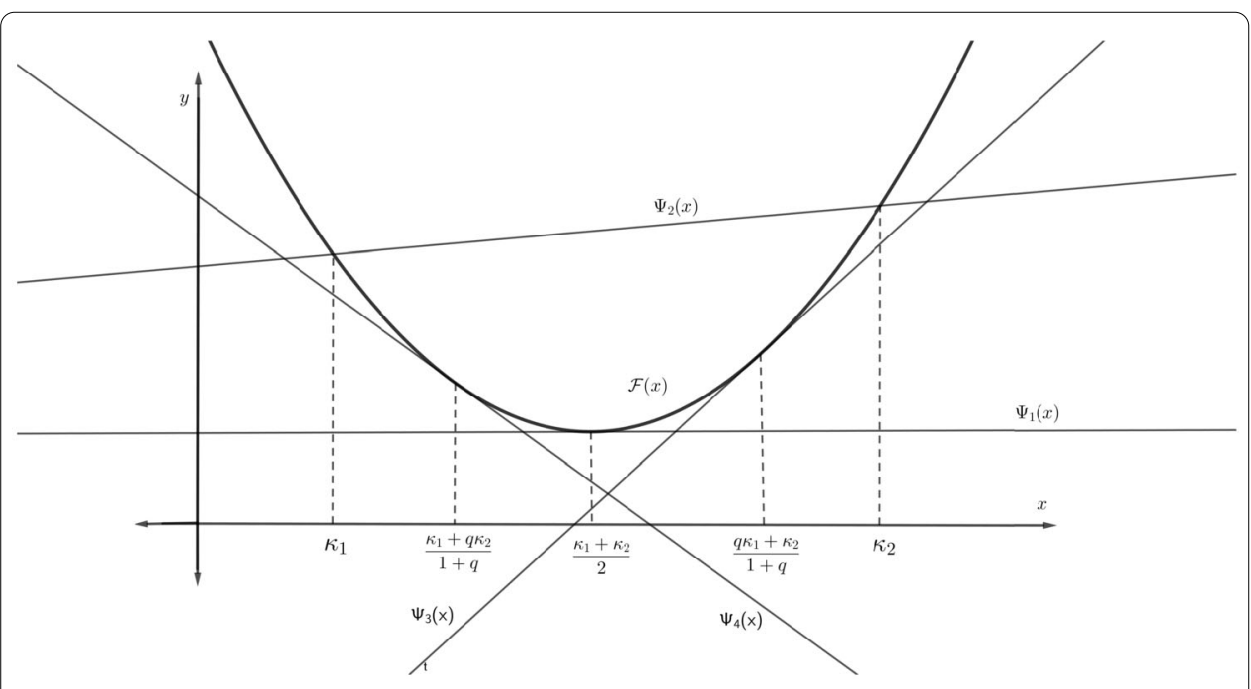

Figure 2 Graphs of a convex function $\mathcal{F}$ and some tangent lines 


$$
\begin{aligned}
= & \left(\kappa_{2}-\kappa_{1}\right) \mathcal{F}\left(\frac{\kappa_{1}+\kappa_{2}}{2}\right)-\mathcal{F}^{\prime}\left(\frac{\kappa_{1}+\kappa_{2}}{2}\right) \\
& \times\left[\left.\left(\frac{1-q}{1-q^{2}}\right)\left(\frac{1+q}{2}\right)\left(\kappa_{2}-\varkappa\right)^{2}\right|_{\kappa_{1}} ^{\kappa_{2}}+\frac{\kappa_{1}-\kappa_{2}}{2}\left(\kappa_{2}-\kappa_{1}\right)\right] \\
= & \left(\kappa_{2}-\kappa_{1}\right) \mathcal{F}\left(\frac{\kappa_{1}+\kappa_{2}}{2}\right)-\mathcal{F}^{\prime}\left(\frac{\kappa_{1}+\kappa_{2}}{2}\right)\left[\frac{\left(\kappa_{2}-\kappa_{1}\right)^{2}}{2}-\frac{\left(\kappa_{2}-\kappa_{1}\right)^{2}}{2}\right] \\
= & \left(\kappa_{2}-\kappa_{1}\right) \mathcal{F}\left(\frac{\kappa_{1}+\kappa_{2}}{2}\right) .
\end{aligned}
$$

This gives the proof of the first inequality in (4.1).

On the other hand, we have the function $\Psi_{2}(\varkappa)=\mathcal{F}\left(\kappa_{2}\right)+\frac{\mathcal{F}\left(\kappa_{2}\right)-\mathcal{F}\left(\kappa_{1}\right)}{\kappa_{2}-\kappa_{1}}\left(\varkappa-\kappa_{2}\right)$ (see Fig. 2). Since $\mathcal{F}$ is a convex function on $\left[\kappa_{1}, \kappa_{2}\right]$, we have the inequality

$$
\mathcal{F}(\varkappa) \leq \Psi_{2}(\varkappa)
$$

and thus, by Theorem 10, we get

$$
\int_{\kappa_{1}}^{\kappa_{2}} \mathcal{F}(\varkappa)^{\kappa_{2}} d_{q}^{T} \varkappa \leq \int_{\kappa_{1}}^{\kappa_{2}} \Psi_{2}(\varkappa)^{\kappa_{2}} d_{q}^{T} \varkappa
$$

for all $\varkappa \in\left[\kappa_{1}, \kappa_{2}\right]$. By the definition of ${ }^{\kappa_{2}} T_{q}$-integral, we have

$$
\begin{aligned}
& \int_{\kappa_{1}}^{\kappa_{2}} \Psi_{2}(\varkappa)^{\kappa_{2}} d_{q}^{T} \varkappa \\
& \quad=\int_{\kappa_{1}}^{\kappa_{2}}\left(\mathcal{F}\left(\kappa_{2}\right)+\frac{\mathcal{F}\left(\kappa_{2}\right)-\mathcal{F}\left(\kappa_{1}\right)}{\kappa_{2}-\kappa_{1}}\left(\varkappa-\kappa_{2}\right)\right) \kappa^{\kappa_{2}} d_{q}^{T} \varkappa \\
& \quad=\left(\kappa_{2}-\kappa_{1}\right) \mathcal{F}\left(\kappa_{2}\right)-\frac{\mathcal{F}\left(\kappa_{2}\right)-\mathcal{F}\left(\kappa_{1}\right)}{\kappa_{2}-\kappa_{1}} \int_{\kappa_{1}}^{\kappa_{2}}\left(\kappa_{2}-\varkappa\right)^{\kappa_{2}} d_{q}^{T} \varkappa \\
& =\left(\kappa_{2}-\kappa_{1}\right) \mathcal{F}\left(\kappa_{2}\right)-\frac{\mathcal{F}\left(\kappa_{2}\right)-\mathcal{F}\left(\kappa_{1}\right)}{\kappa_{2}-\kappa_{1}}\left(\frac{1-q}{1-q^{2}}\right)\left(\frac{1+q}{2}\right)\left(\kappa_{2}-\kappa_{1}\right)^{2} \\
& =\left(\kappa_{2}-\kappa_{1}\right) \mathcal{F}\left(\kappa_{2}\right)-\frac{\mathcal{F}\left(\kappa_{2}\right)-\mathcal{F}\left(\kappa_{1}\right)}{\kappa_{2}-\kappa_{1}} \frac{\left(\kappa_{2}-\kappa_{1}\right)^{2}}{2} \\
& =\left(\kappa_{2}-\kappa_{1}\right) \frac{\mathcal{F}\left(\kappa_{1}\right)+\mathcal{F}\left(\kappa_{2}\right)}{2} .
\end{aligned}
$$

The proof is completed.

Remark 1 In Theorem 11, if we take the limit $q \rightarrow 1^{-}$, we recapture the classical HermiteHadamard inequality for convex function.

Theorem 12 Let $\mathcal{F}:\left[\kappa_{1}, \kappa_{2}\right] \rightarrow \mathbb{R}$ be a convex differentiable function on $\left[\kappa_{1}, \kappa_{2}\right]$ and $0<$ $q<1$. Then we have

$$
\begin{aligned}
\mathcal{F}\left(\frac{q \kappa_{1}+\kappa_{2}}{1+q}\right)+\frac{q-1}{1+q} \frac{\left(\kappa_{2}-\kappa_{1}\right)}{2} \mathcal{F}^{\prime}\left(\frac{q \kappa_{1}+\kappa_{2}}{1+q}\right) & \leq \frac{1}{\kappa_{2}-\kappa_{1}} \int_{\kappa_{1}}^{\kappa_{2}} \mathcal{F}(\varkappa)^{\kappa_{2}} d_{q}^{T} \varkappa \\
& \leq \frac{\mathcal{F}\left(\kappa_{1}\right)+\mathcal{F}\left(\kappa_{2}\right)}{2} .
\end{aligned}
$$


Proof Since $\mathcal{F}$ is a differentiable function on $\left[\kappa_{1}, \kappa_{2}\right]$, there is a tangent line for the function $\mathcal{F}$ at the point $\frac{q \kappa_{1}+\kappa_{2}}{1+q} \in\left(\kappa_{1}, \kappa_{2}\right)$. This tangent line can be expressed as a function $\Psi_{3}(\varkappa)=$ $\mathcal{F}\left(\frac{q \kappa_{1}+\kappa_{2}}{1+q}\right)+\mathcal{F}^{\prime}\left(\frac{q \kappa_{1}+\kappa_{2}}{1+q}\right)\left(\varkappa-\frac{q \kappa_{1}+\kappa_{2}}{1+q}\right)$. Since $\mathcal{F}$ is a convex function on $\left[\kappa_{1}, \kappa_{2}\right]$, then we have the following inequality:

$$
\Psi_{3}(\varkappa) \leq \mathcal{F}(\varkappa)
$$

for all $\varkappa \in\left[\kappa_{1}, \kappa_{2}\right]$ (see Fig. 2). From Theorem 10, we have

$$
\int_{\kappa_{1}}^{\kappa_{2}} \Psi_{3}(\varkappa)^{\kappa_{2}} d_{q}^{T} \varkappa \leq \int_{\kappa_{1}}^{\kappa_{2}} \mathcal{F}(\varkappa)^{\kappa_{2}} d_{q}^{T} \varkappa
$$

By definition of ${ }^{\kappa_{2}} T_{q}$-integral, we get

$$
\begin{aligned}
\int_{\kappa_{1}}^{\kappa_{2}} & \Psi_{3}(\varkappa)^{\kappa_{2}} d_{q}^{T} \varkappa \\
= & \int_{\kappa_{1}}^{\kappa_{2}}\left[\mathcal{F}\left(\frac{q \kappa_{1}+\kappa_{2}}{1+q}\right)+\mathcal{F}^{\prime}\left(\frac{q \kappa_{1}+\kappa_{2}}{1+q}\right)\left(\varkappa-\frac{q \kappa_{1}+\kappa_{2}}{1+q}\right)\right] \kappa_{2} d_{q}^{T} \varkappa \\
= & \left(\kappa_{2}-\kappa_{1}\right) \mathcal{F}\left(\frac{q \kappa_{1}+\kappa_{2}}{1+q}\right)-\mathcal{F}^{\prime}\left(\frac{q \kappa_{1}+\kappa_{2}}{1+q}\right)\left[\int_{\kappa_{1}}^{\kappa_{2}}\left(\kappa_{2}-\varkappa+q \frac{\kappa_{1}-\kappa_{2}}{1+q}\right) \kappa_{2} d_{q}^{T} \varkappa\right] \\
= & \left(\kappa_{2}-\kappa_{1}\right) \mathcal{F}\left(\frac{q \kappa_{1}+\kappa_{2}}{1+q}\right) \\
& -\mathcal{F}^{\prime}\left(\frac{q \kappa_{1}+\kappa_{2}}{1+q}\right)\left[\left(\frac{1-q}{1-q^{2}}\right)\left(\frac{1+q}{2}\right)\left(\kappa_{2}-\kappa_{1}\right)^{2}+\frac{\kappa_{1}-\kappa_{2}}{1+q} q\left(\kappa_{2}-\kappa_{1}\right)\right] \\
= & \left(\kappa_{2}-\kappa_{1}\right) \mathcal{F}\left(\frac{q \kappa_{1}+\kappa_{2}}{1+q}\right)-\mathcal{F}^{\prime}\left(\frac{q \kappa_{1}+\kappa_{2}}{1+q}\right)\left[\frac{\left(\kappa_{2}-\kappa_{1}\right)^{2}}{2}-\frac{q\left(\kappa_{2}-\kappa_{1}\right)^{2}}{1+q}\right],
\end{aligned}
$$

which completes the proof.

Theorem 13 Let $\mathcal{F}:\left[\kappa_{1}, \kappa_{2}\right] \rightarrow \mathbb{R}$ be a convex differentiable function on $\left[\kappa_{1}, \kappa_{2}\right]$ and $0<$ $q<1$. Then we have

$$
\begin{aligned}
\mathcal{F}\left(\frac{\kappa_{1}+q \kappa_{2}}{1+q}\right)+\frac{1-q}{1+q} \frac{\left(\kappa_{2}-\kappa_{1}\right)}{2} \mathcal{F}^{\prime}\left(\frac{\kappa_{1}+q \kappa_{2}}{1+q}\right) & \leq \frac{1}{\kappa_{2}-\kappa_{1}} \int_{\kappa_{1}}^{\kappa_{2}} \mathcal{F}(\varkappa)^{\kappa_{2}} d_{q}^{T} \varkappa \\
& \leq \frac{\mathcal{F}\left(\kappa_{1}\right)+\mathcal{F}\left(\kappa_{2}\right)}{2} .
\end{aligned}
$$

Proof Since $\mathcal{F}$ is a differentiable function on $\left[\kappa_{1}, \kappa_{2}\right]$, there is a tangent line for the function $\mathcal{F}$ at the point $\frac{\kappa_{1}+q \kappa_{2}}{1+q} \in\left(\kappa_{1}, \kappa_{2}\right)$. This tangent line can be expressed as a function $\Psi_{4}(\varkappa)=$ $\mathcal{F}\left(\frac{\kappa_{1}+q \kappa_{2}}{1+q}\right)+\mathcal{F}^{\prime}\left(\frac{\kappa_{1}+q \kappa_{2}}{1+q}\right)\left(\varkappa-\frac{\kappa_{1}+q \kappa_{2}}{1+q}\right)$. Since $\mathcal{F}$ is a convex function on $\left[\kappa_{1}, \kappa_{2}\right]$, then we have the following inequality:

$$
\Psi_{4}(\varkappa) \leq \mathcal{F}(\varkappa)
$$

for all $\varkappa \in\left[\kappa_{1}, \kappa_{2}\right]$ (see Fig. 2). By Theorem 10, we have

$$
\int_{\kappa_{1}}^{\kappa_{2}} \Psi_{4}(\varkappa)^{\kappa_{2}} d_{q}^{T} \varkappa \leq \int_{\kappa_{1}}^{\kappa_{2}} \mathcal{F}(\varkappa)^{\kappa_{2}} d_{q}^{T} \varkappa
$$


By definition of ${ }^{\kappa_{2}} T_{q}$-integral, we get

$$
\begin{aligned}
\int_{\kappa_{1}}^{\kappa_{2}} & \Psi_{4}(\varkappa)^{\kappa_{2}} d_{q}^{T} \varkappa \\
= & \int_{\kappa_{1}}^{\kappa_{2}}\left[\mathcal{F}\left(\frac{\kappa_{1}+q \kappa_{2}}{1+q}\right)+\mathcal{F}^{\prime}\left(\frac{\kappa_{1}+q \kappa_{2}}{1+q}\right)\left(\varkappa-\frac{\kappa_{1}+q \kappa_{2}}{1+q}\right)\right] \kappa_{2} d_{q}^{T} \varkappa \\
= & \left(\kappa_{2}-\kappa_{1}\right) \mathcal{F}\left(\frac{\kappa_{1}+q \kappa_{2}}{1+q}\right)-\mathcal{F}^{\prime}\left(\frac{\kappa_{1}+q \kappa_{2}}{1+q}\right) \int_{\kappa_{1}}^{\kappa_{2}}\left(\kappa_{2}-\varkappa+\frac{\kappa_{1}-\kappa_{2}}{1+q}\right) \kappa_{2} d_{q}^{T} \varkappa \\
= & \left(\kappa_{2}-\kappa_{1}\right) \mathcal{F}\left(\frac{\kappa_{1}+q \kappa_{2}}{1+q}\right) \\
& -\mathcal{F}^{\prime}\left(\frac{\kappa_{1}+q \kappa_{2}}{1+q}\right)\left[\left(\frac{1-q}{1-q^{2}}\right)\left(\frac{1+q}{2}\right)\left(\kappa_{2}-\kappa_{1}\right)^{2}+\left(\kappa_{2}-\kappa_{1}\right) \frac{\kappa_{1}-\kappa_{2}}{1+q}\right] \\
= & \left(\kappa_{2}-\kappa_{1}\right) \mathcal{F}\left(\frac{\kappa_{1}+q \kappa_{2}}{1+q}\right)-\mathcal{F}^{\prime}\left(\frac{\kappa_{1}+q \kappa_{2}}{1+q}\right)\left[\frac{\left(\kappa_{2}-\kappa_{1}\right)^{2}}{2}-\frac{\left(\kappa_{2}-\kappa_{1}\right)^{2}}{1+q}\right] .
\end{aligned}
$$

This gives the proof of the theorem.

Theorem 14 Let $\mathcal{F}:\left[\kappa_{1}, \kappa_{2}\right] \rightarrow \mathbb{R}$ be a convex differentiable function on $\left[\kappa_{1}, \kappa_{2}\right]$ and $0<$ $q<1$. Then we have

$$
\max \left\{I_{1}, I_{2}, I_{3}\right\} \leq \frac{1}{\kappa_{2}-\kappa_{1}} \int_{\kappa_{1}}^{\kappa_{2}} \mathcal{F}(\varkappa)^{\kappa_{2}} d_{q}^{T} \varkappa \leq \frac{\mathcal{F}\left(\kappa_{1}\right)+\mathcal{F}\left(\kappa_{2}\right)}{2}
$$

where

$$
\begin{aligned}
& I_{1}=\mathcal{F}\left(\frac{\kappa_{1}+\kappa_{2}}{2}\right), \\
& I_{2}=\mathcal{F}\left(\frac{q \kappa_{1}+\kappa_{2}}{1+q}\right)+\frac{q-1}{1+q} \frac{\left(\kappa_{2}-\kappa_{1}\right)}{2} \mathcal{F}^{\prime}\left(\frac{q \kappa_{1}+\kappa_{2}}{1+q}\right) \\
& I_{3}=\mathcal{F}\left(\frac{\kappa_{1}+q \kappa_{2}}{1+q}\right)+\frac{1-q}{1+q} \frac{\left(\kappa_{2}-\kappa_{1}\right)}{2} \mathcal{F}^{\prime}\left(\frac{\kappa_{1}+q \kappa_{2}}{1+q}\right) .
\end{aligned}
$$

Proof A combination of (4.1), (4.3), and (4.5) gives (4.7) and the proof is completed.

\section{Conclusion}

In this article, we proved a new idea of quantum integrals which is called ${ }^{\kappa_{2}} T_{q}$-integral. By using this idea, we proved several properties for quantum integrals. Further, we presented several Hermite-Hadamard type ${ }^{\kappa_{2}} T_{q}$-integral inequalities within a class of convexity. It is also shown that some classical results can be obtained by the results presented in the current research by taking the limit $q \rightarrow 1^{-}$. It will be an interesting problem to prove similar inequalities for the functions of two variables.

\section{Acknowledgements}

The authors would like to express their sincere thanks to the editor and the anonymous reviewers for their helpful comments and suggestions.

Funding

There is no funding for this work. 
Availability of data and materials

Not applicable.

\section{Declarations}

\section{Competing interests}

The authors declare that they have no competing interests.

\section{Authors' contributions}

All authors contributed equally to the writing of this paper. All authors read and approved the final manuscript.

\section{Author details}

'Department of Mathematics, Faculty of Science and Arts, Düzce University, Düzce, Turkey. ${ }^{2}$ Department of Mathematical, Zhejiang Normal University, Jinhua 321004, China.

\section{Publisher's Note}

Springer Nature remains neutral with regard to jurisdictional claims in published maps and institutional affiliations.

Received: 11 March 2021 Accepted: 20 October 2021 Published online: 30 October 2021

\section{References}

1. Alp, N., Sariakaya, M.Z:: A new definition and properties of quantum integral which calls $\bar{q}$-integral. Konuralp J. Math. 5(2), 146-159 (2017)

2. Alp, N., Sarikaya, M.Z:: Hermite Hadamard's type inequalities for co-ordinated convex functions on quantum integral. Appl. Math. E-Notes 20, 341-356 (2020)

3. Alp, N., Sarikaya, M.Z., Kunt, M., Iscan, l.: q-Hermite Hadamard inequalities and quantum estimates for midpoint type inequalities via convex and quasi-convex functions. J. King Saud Univ., Sci. 30, 193-203 (2018)

4. Belarbi, S., Dahmani, Z.: On some new fractional integral inequalities. JIPAM. J. Inequal. Pure Appl. Math. 10, Article ID 86 (2009)

5. Bermudo, S., Kórus, P., Valdés, J.N.: On q-Hermite-Hadamard inequalities for general convex functions. Acta Math. Hung. 162, 364-374 (2020)

6. Dahmani, Z: New inequalities in fractional integrals. Int. J. Nonlinear Sci. 9, 493-497 (2010)

7. Ernst, T.: The History of Q-Calculus and New Method. Department of Mathematics, Uppsala University, Sweden (2000)

8. Ernst, T.: A method for q-calculus. J. Nonlinear Math. Phys. 10(4), 487-525 (2003)

9. Ernst, T: A Comprehensive Treatment of $q$-Calculus. Springer, Basel (2012)

10. Gauchman, H.: Integral inequalities in q-calculus. Comput. Math. Appl. 47, 281-300 (2004). https://doi.org/10.1016/S0898-1221(04)90025-9

11. Hadamard, J.: Etude sur les propriétés des fonctions entiéres et en particulier dune fonction considerèe par Riemann. J. Math. Pures Appl. 58,171-215 (1893)

12. Jackson, F.H.: On a q-definite integrals. Q. J. Pure Appl. Math. 41, 193-203 (1910)

13. Kac, V., Cheung, P.: Quantum Calculus. Springer, New York (2002)

14. Noor, M., Noor, K., Awan, M.: Quantum Ostrowski inequalities for q-differentiable convex functions. J. Math. Inequal. 10, 1013-1018 (2016)

15. Noor, M.A., Noor, K.I., Awan, M.U.: Some quantum estimates for Hermite-Hadamard inequalities. Appl. Math. Comput. 251, 675-679 (2015)

16. Noor, M.A., Noor, K.I., Awan, M.U.: Some quantum integral inequalities via preinvex functions. Appl. Math. Comput. 269, 242-251 (2015)

17. Ogunmez, H., Ozkan, U.M.: Fractional quantum integral inequalities. J. Inequal. Appl. 2011, Article ID 787939 (2011)

18. Sudsutad, W., Ntouyas, S.K., Tariboon, J.: Quantum integral inequalities for convex functions. J. Math. Inequal. 9(3), 781-793 (2015)

19. Tariboon, J., Ntouyas, S.K.: Quantum calculus on finite intervals and applications to impulsive difference equations. Adv. Differ. Equ. 2013, 282 (2013)

20. Tariboon, J., Ntouyas, S.K.: Quantum integral inequalities on finite intervals. J. Inequal. Appl. 2014, 121 (2014)

21. Zhuang, H., Liu, W., Park, J.: Some quantum estimates of Hermite-Hadamard inequalities for quasi-convex functions. Mathematics 7, 152 (2019) 